\title{
Consumo de sustancias y suicidios en México: resultados del Sistema de Vigilancia Epidemiológica de las Adicciones, 1994-2006
}

\author{
René Ocampo, Psiq, (I) letza Bojorquez, Dr epidemio,, (I) Mario Cortés, MCS.(I)
}

\author{
Ocampo R, Bojórquez I, Cortés M. \\ Consumo de sustancias y suicidios en \\ México: resultados del Sistema de Vigilancia \\ Epidemiológica de las Adicciones, 1994-2006. \\ Salud Publica Mex 2009;5 I:306-313.
}

\begin{abstract}
Resumen
Objetivo. Determinar la relación que existe entre el consumo y número de sustancias y la presentación del suicidio. Material y métodos. Los datos se tomaron de la cédula forense del Sistema de Vigilancia Epidemiológica de las Adicciones entre 1994 y 2006 de 27 entidades federativas participantes en México. Resultados. El suicidio se presentó en $8.7 \%$ de las defunciones por causa violenta en el periodo de estudio. En los hombres se observó que a medida que aumentaba el número de sustancias se elevaba la posibilidad para fallecer por suicidio, en comparación con los decesos por otras causas (una sustancia: $R M=1.8$; dos o más: $R M=3.3$ ). En las mujeres, dicha posibilidad se mantiene prácticamente igual en relación con el aumento del número de sustancias detectadas (una sustancia: $R M=3.2$; dos o más: $R M=3.6$ ). Conclusión. El consumo de sustancias es un factor importante vinculado con el suicidio en los sujetos cuya causa de defunción fue dictaminada por el Servicio Médico Forense mexicano.
\end{abstract}

Palabras clave: suicidio; drogas ilícitas; epidemiología; psiquiatría forense; México
Ocampo R, Bojórquez I, Cortés M.

Substance use in suicides in

Mexico: results of the Epidemiological Surveillance

System of Addictions, 1994-2006.

Salud Publica Mex 2009;51:306-313.

\section{Abstract}

Objective. To determine the association between substance use and the number of substances with the presentation of suicide. Material and Methods. Data were taken from the forensic certificate of the Epidemiological Surveillance System of Addictions in the period between 1994 and 2006 from 27 states in Mexico. Results. Suicide was detected in $8.7 \%$ of the violent deaths during the study period.Among men, it was observed that the increased number of substances increased the possibility for death by suicide, compared to deaths from other causes (one substance: $O R=1.8$; two or more: $O R=$ 3.3). In women, that possibility remains virtually unchanged with the increase in the number of substances detected (one substance: $O R=3.2$; two or more: $O R=3.6$ ). Discussion. The use of substances is a major factor associated with suicide in the population whose cause of death was issued by the Mexican Forensic Medical Services.

Keywords: suicide; street drugs; epidemiology; forensic psychiatry; Mexico 
E suicidio en los últimos años ha captado la atención de las autoridades de salud e investigadores debido al aumento de sus tasas a nivel mundial. En términos operacionales, el suicidio se define como la muerte consecutiva a un daño autoinfligido de manera intencional, en consideración de tres componentes: la muerte por daño, la conducta contra sí mismo y la intencionalidad. ${ }^{1}$ El concepto del suicidio es complejo y ha evolucionado desde su enfoque de un acto aislado hasta su inclusión en un concepto más amplio que comprende un continuo entre la ideación suicida, la planeación suicida, el intento suicida y el suicidio consumado. ${ }^{2}$ Según cifras de la OMS, la tasa mundial de suicidio en el año 2000 se estimó en 16 por cada 100000 habitantes, mientras que la tasa de suicido en la población general en México hasta el 2003 era de 4 por cada 100000 habitantes. ${ }^{3}$ De acuerdo con cifras oficiales del Instituto Nacional de Estadística, Geografía e Informática (INEGI), ${ }^{4}$ en México el suicidio representó en el año 2006 una proporción de $7.9 \%$ de las muertes por causa violenta $(8.4 \%$ en hombres y $6.3 \%$ en mujeres).

Entre los factores de riesgo para la conducta suicida, el que ha recibido más atención en los estudios epidemiológicos es la presencia de trastornos mentales, ya que se ha observado que 90 a $98 \%$ de las personas que intentan suicidarse padecía algún trastorno mental en el eje I, de acuerdo con el Manual Diagnóstico y Estadístico de los Trastornos Mentales (DSM) y 46\% algún trastorno de la personalidad, en particular los trastornos límite, antisocial y obsesivo-compulsivo. ${ }^{5-12}$ Entre los trastornos del eje I destacan el trastorno depresivo mayor y el trastorno por consumo de sustancias. Con respecto a este último, el riesgo para presentar la conducta suicida en la población con tal padecimiento se ha calculado en 2.6 veces el de los sujetos que no lo tienen, incluso al controlar de forma estadística por los trastornos psiquiátricos comórbidos. ${ }^{9,13}$

Entre las sustancias cuyo uso se reporta con mayor frecuencia en los individuos con conducta suicida figuran el alcohol, cannabis, sedantes, estimulantes y opioides, aunque se ha considerado que no es el tipo de sustancias consumidas sino el número de ellas lo que incrementa el riesgo de suicidio. ${ }^{9}$ De manera general, dichos sujetos refieren más síntomas depresivos y comorbilidad psiquiátrica que la población general, ${ }^{14}$ utilizan fármacos de prescripción como método suicida (hasta $50 \%$ ) y experimentan un mayor número de sobredosis previas. ${ }^{15-17}$ También en los estudios epidemiológicos se ha encontrado que el consumo actual de sustancias predice por sí sólo el comportamiento suicida de manera consistente. $^{9}$

El siguiente trabajo se presenta a partir de información obtenida de la base de datos sobre mortalidad y consumo de sustancias en individuos registrados por el Servicio Médico Forense (SEMEFO) y notificados al Sistema de Vigilancia Epidemiológica de las Adicciones (SISVEA) en México. Los objetivos del estudio son: a) describir el consumo de sustancias en personas que fallecieron por suicidio y $b$ ) determinar la relación que existe entre el consumo y número de sustancias y la perpetración del suicidio.

\section{Material y métodos}

La información que se presenta en el presente trabajo se tomó de manera transversal de la base de datos sobre defunciones por causa violenta que el Servicio Médico Forense de las entidades federativas participantes informa al SISVEA de la Dirección General Adjunta de Epidemiología (DGAE), la cual abarca el periodo comprendido entre 1994 y 2006. Dicho periodo corresponde al tiempo en que el SISVEA ha estado en operación. ${ }^{18}$

Durante el periodo de operación del SISVEA han participado 27 entidades federativas. A continuación se enlistan las entidades y los años en los que han participado en dicho sistema: Aguascalientes (1997-2006), Baja California (1994-2006), Baja California Sur (2003 y 2004), Campeche (2003-2006), Chiapas (2003-2005), Coahuila (1994-1995 y 2005-2006), Colima (2005 y 2006), Chihuahua (1994-1999 y 2001-2006), Durango (19952006), Guanajuato (1994-2006), Guerrero (2003-2006), Hidalgo (2002-2006), Jalisco (1994-2006), Estado de México (2003-2006), Puebla (2004-2006), Morelos (2005 y 2006), Querétaro (1999 y 2001-2006), Quintana Roo (2000-2006), Oaxaca (2002), San Luis Potosí (1994-2006), Tlaxcala (2005-2006), Sinaloa (1994-2002), Sonora (2002), Tamaulipas (1994-2001), Veracruz (2003-2006), Yucatán (1994-1995 y 1997-2006) y Zacatecas (2002-2006).

La base de datos ya mencionada se elaboró a partir de la información recabada en la cédula forense del SISVEA. Dicha cédula incluye características sociodemográficas (edad, sexo, escolaridad y estado civil), condiciones del fallecimiento, causa de muerte (intoxicación, asfixia, arma de fuego, etc.), lugar donde ocurrió la muerte, intención (accidente, suicidio, homicidio y muerte súbita), ocurrencia de la muerte bajo la influencia de alguna sustancia y el tipo de sustancia detectada.

En dicha cédula, la "muerte bajo influencia de alguna sustancia" la define el médico forense, quien registra el tipo de sustancia que se relacionó con la muerte, tras revisar los nombres comerciales si se trata de medicamentos y las diferentes variantes de los fármacos médicos. ${ }^{18}$ En la cédula forense del SISVEA es posible registrar más de una sustancia, sin describir el 
patrón de consumo del sujeto o realizar un diagnóstico de trastorno por consumo de sustancias.

El análisis de los datos se llevó a cabo con el programa STATA, versión 9. Los datos se presentan en porcentajes. Para fines del análisis, la intención de la muerte se reagrupó en dos categorías, "suicidio" y "defunción por otra causa". Esta última incluye a las muertes por homicidio, accidentes y muerte súbita. Se calcularon las razones de momios (RM) e intervalos de confianza al 95\% para conocer la relación entre las variables sociodemográficas y el consumo de sustancias con el suicidio, tras tomar como categorías de referencia a las variables con menor frecuencia. Dado que al estratificar por sexo se encontraron resultados heterogéneos en estas asociaciones, se decidió presentar los resultados en hombres y mujeres por separado. Para determinar la relación tanto del consumo de sustancias como del número de éstas con la presentación del suicidio se utilizó el análisis de regresión logística y se controló de manera estadística por las variables edad, estado civil y escolaridad.

\section{Resultados}

\section{Mortalidad en SEMEFO}

El total de defunciones registradas en la cédula forense del SISVEA de 1994 a 2006 fue de 101 172. La distribución por sexo, escolaridad y estado civil se muestra en el cuadro I. De las cuatro causas de muerte reportadas en la cédula de SEMEFO de 1994 a 2006, el suicidio ocupó la cuarta posición con $8.7 \%$ de los casos.

\section{Variables sociodemográficas y suicidio}

De manera general, el suicidio fue más frecuente en los hombres $(9.1 \%)$ que en las mujeres $(7 \%)$. Las defunciones por suicidio también fueron más frecuentes en el grupo de edad de 29 a 39 años (28\%), en el grupo con escolaridad hasta secundaria (13.4\%) y en los solteros (10.6\%) (cuadro II).

Las relaciones entre las variables sociodemográficas, el consumo de sustancias y el suicidio se presentan de acuerdo con el sexo en el cuadro III. Tanto en los hombres como en las mujeres, y al comparar con las defunciones por otra causa, los momios fueron mayores para el grupo de edad de 20 a 39 años (hombres: $R M=$ 2.48; IC95\%, 2.34-2.62; mujeres: $R M=3.84$; IC95\%, 3.364.40 , al tomar como grupo de referencia a los mayores de 40 años), en el grupo con escolaridad de secundaria o mayor (hombres: $R M=1.16$; IC95\%, 1.08-1.25; mujeres: $R M=1.91$; IC95\%, 1.64-2.22, al tomar como referencia a los sujetos con primaria o menor) y en los solteros
Cuadro I

\section{DistRIBUCIÓN SOCIODEMOGRÁFICA DE LA MUESTRA del Servicio Médico Forense 1994-2006}

\begin{tabular}{lcc}
$\begin{array}{l}\text { Sexo } \\
\text { Masculino }\end{array}$ & $N$ & $\%$ \\
\hline Femenino & 80714 & 80.1 \\
\hline Total* & 19990 & 19.9 \\
$\begin{array}{l}\text { Edad } \\
5-9\end{array}$ & 100704 & 100 \\
\hline $10-14$ & 2005 & \\
\hline $15-19$ & 2812 & 2.1 \\
\hline $20-24$ & 7826 & 8.1 \\
\hline $25-29$ & 10320 & 10.6 \\
\hline $30-34$ & 9792 & 10.1 \\
\hline $35-39$ & 8975 & 9.2 \\
\hline 40 o mayores & 8378 & 8.6 \\
\hline Tota* & 47104 & 48 \\
\hline
\end{tabular}

Escolaridad

\begin{tabular}{lrr} 
Primaria o menos & 49102 & 65.9 \\
\hline Secundaria & 14316 & 19.2 \\
\hline Preparatoria & 6594 & 8.8 \\
\hline Licenciatura o posgrado & 4405 & 5.9 \\
\hline Total* & 74417 & 100 \\
& & \\
$\begin{array}{l}\text { Estado civil } \\
\quad \text { Casado/unión libre }\end{array}$ & 39102 & 49.9 \\
\hline Divorciado/separado/viudo & 9961 & 12.7 \\
\hline Soltero & 29303 & 37.4 \\
\hline Total* & 78336 & 100
\end{tabular}

Fuente: Sistema Nacional de Vigilancia Epidemiológica de las Adicciones, México, 1994-2006

* Existe variación en los totales debido a los datos perdidos

(hombres: $R M=1.99$; IC95\%, 1.77-2.24; mujeres: $R M=$ 3.23; IC95\%, 2.62-3.98, al tomar como referencia al grupo "separado/divorciado/viudo") (cuadro III).

\section{Suicidio bajo la influencia de sustancias}

De acuerdo con el reporte del SEMEFO, la principal sustancia detectada en los casos de suicidio fue el alcohol $(72.9 \%)$, seguida por el grupo de los estimulantes $(9.6 \%)$ y el grupo de los sedantes (6.8\%).

En el análisis estratificado por sexo se encontró que tanto en hombres como en mujeres la posibilidad de estar bajo la influencia de sustancias fue mayor en los fallecimientos por suicidio, en comparación con quienes 


\section{Cuadro II}

Variables sociodemográficas por causa de muerte, Servicio Médico Forense I994-2006

\begin{tabular}{|c|c|c|c|c|c|}
\hline \multirow[t]{2}{*}{ Causa de defunción } & $\begin{array}{c}\text { Accidentes } \\
\%\end{array}$ & $\begin{array}{c}\text { Muerte súbita } \\
\%\end{array}$ & $\begin{array}{c}\text { Homicidio } \\
\%\end{array}$ & $\begin{array}{c}\text { Suicidio } \\
\%\end{array}$ & $\begin{array}{l}\text { TOTAL } \\
N(\%)\end{array}$ \\
\hline & 47.1 & 25.4 & 18.9 & 8.7 & $98434(100)$ \\
\hline \multicolumn{6}{|l|}{ Sexo } \\
\hline Masculino & 47 & 23.3 & 20.6 & 9.1 & $78552(100)$ \\
\hline Femenino & 47.4 & 34.1 & 11.6 & 7 & $19465(100)$ \\
\hline \multicolumn{6}{|l|}{ Edad } \\
\hline $5-9$ & 73.8 & 12.2 & 12.7 & 1.3 & I $953(100)$ \\
\hline $10-14$ & 66.3 & 10.1 & 14.6 & 9 & $2765(100)$ \\
\hline $15-19$ & 53.7 & 6.7 & 25.5 & 14.1 & $7670(100)$ \\
\hline $20-24$ & 50.3 & 7.2 & 27.6 & 14.8 & $10102(100)$ \\
\hline $25-29$ & 48.2 & 11.2 & 27.3 & 13.3 & $9557(100)$ \\
\hline $30-34$ & 46.3 & 14.3 & 26.8 & 12.7 & $8739(100)$ \\
\hline $35-39$ & 46 & 19.2 & 24.3 & 10.5 & $8174(100)$ \\
\hline 40 y mayores & 43.1 & 38.9 & 12.8 & 5.2 & $45745(100)$ \\
\hline \multicolumn{6}{|l|}{ Escolaridad } \\
\hline Primaria o menos & 45.9 & 30.3 & 16.3 & 7.5 & $47965(100)$ \\
\hline Secundaria & 51 & 14.5 & 21 & 13.4 & $13996(100)$ \\
\hline Preparatoria & 54.6 & 14.1 & 20 & 11.4 & $6504(100)$ \\
\hline Licenciatura/posgrado & 52.3 & 19.2 & 18 & 10.5 & $4321(100)$ \\
\hline \multicolumn{6}{|l|}{ Estado civil } \\
\hline Casado/unión libre & 47.3 & 24.5 & 19.1 & 9.1 & $38289(100)$ \\
\hline Divorciado / Viudo/separado & 41.4 & 44.3 & 9.5 & 4.7 & $9741(100)$ \\
\hline Soltero & 51.8 & 19 & 18.6 & 10.6 & $28630(100)$ \\
\hline
\end{tabular}

Fuente: Sistema Nacional de Vigilancia Epidemiológica de las Adicciones, México, 1994-2006

murieron por otras causas; empero, dicha posibilidad fue mayor para las mujeres $(R M=3.94, I C 95 \%, 3.40-4.57$ en mujeres; $R M=1.96, I C 95 \%$, 1.86-2.07 en hombres). En cuanto al número de sustancias, la posibilidad de fallecer por suicidio se incrementó en los casos de suicidio a medida que aumentó el número de sustancias detectadas tanto en hombres como en mujeres, al comparar con los fallecimientos por otras causas. De nueva cuenta, esta posibilidad fue mayor en las mujeres (cuadro III).

Para observar la relación independiente de las variables sociodemográficas y el número de sustancias detectadas con la muerte por suicidio, se realizó un análisis de regresión logística en hombres y en mujeres. En el cuadro IV se puede observar que no existieron cambios de consideración en las variables sociodemográficas en los hombres y las mujeres con respecto al análisis bivariado, salvo porque los momios encontrados en la escolaridad ("secundaria o mayor") durante el análisis bivariado ya no fueron significativos en los hombres.

En relación con el número de sustancias, en los hombres se observó que conforme se incrementó el número de sustancias aumentó la posibilidad de fallecer por suicidio (una sustancia: $\mathrm{RM}=1.81$; IC95\%, 1.69-1.93; dos o más sustancias: $\mathrm{RM}=3.30$; IC95\%, 2.79-3.90), en comparación con las muertes por otras causas. En cambio, en las mujeres dicha posibilidad se mantiene prácticamente igual con el aumento del número de sustancias detectadas (una sustancia: $\mathrm{RM}=3.18$; IC95\%, 2.66-3.79; dos o más sustancias: RM=3.61; IC95\%, 1.83-7.11).

\section{Discusión}

Los hallazgos del presente trabajo indican que la detección de sustancias fue más frecuente en los casos de 


\section{Cuadro III}

RELACIÓN ENTRE VARIABLES SOCIODEMOGRÁFICAS, CONSUMO Y NÚMERO DE SUSTANCIAS Y MUERTE POR SUICIDIO EN HOMBRES Y MUJERES, MÉXICO, I 994-2006

\begin{tabular}{|c|c|c|c|c|}
\hline Variable & Hombres & & Mujeres & \\
\hline & $R M$ & IC95\% & $R M$ & IC95\% \\
\hline \multicolumn{5}{|l|}{ Edad } \\
\hline Menor de 20 & $2.01 *$ & $1.86-2.18$ & $3.40 *$ & $2.91-3.98$ \\
\hline 20 a 39 & $2.48 *$ & $2.34-2.62$ & $3.84 *$ & $3.36-4.40$ \\
\hline Mayor de 40 & 1.00 & - & 1.00 & - \\
\hline \multicolumn{5}{|l|}{ Escolaridad } \\
\hline Primaria o menor & 1.00 & - & 1.00 & - \\
\hline Secundaria o mayor & $1.16 *$ & $1.08-1.25$ & $1.91 *$ & $1.64-2.22$ \\
\hline \multicolumn{5}{|l|}{ Estado civil } \\
\hline Soltero & $1.99 *$ & I.77-2.24 & $3.23 *$ & $2.62-3.98$ \\
\hline Casado & $1.64 *$ & $1.46-1.84$ & $2.98 *$ & $2.42-3.66$ \\
\hline Separado/divorciado/viudo & 1.00 & - & 1.00 & - \\
\hline \multicolumn{5}{|l|}{ Bajo influencia de sustancias } \\
\hline Sí & $2.06 *$ & $1.95-2.18$ & $3.94 *$ & $3.40-4.57$ \\
\hline No & 1.00 & - & 1.00 & - \\
\hline \multicolumn{5}{|l|}{ Número de sustancias } \\
\hline Sin sustancias & 1.00 & - & 1.00 & - \\
\hline Una sustancia & $1.96 *$ & $1.86-2.07$ & $3.76^{*}$ & $3.24-4.37$ \\
\hline Dos o más sustancias & $2.70 *$ & $2.36-3.09$ & $4.14 *$ & $2.52-6.81$ \\
\hline$* p<0.05$ & & & & \\
\hline
\end{tabular}

suicidio que en las otras causas de defunción registradas por el SEMEFO y que a un mayor número de sustancias correspondió una mayor probabilidad de muerte por suicidio, tanto en hombres como en mujeres. La sustancia más identificada en los casos de suicidio bajo el influjo de sustancias fue el alcohol.

En cuanto a esta asociación entre el consumo de sustancias y la muerte por suicidio, la mayoría de los estudios nacionales e internacionales indica que la conducta suicida (que incluye desde la ideación hasta el suicidio consumado) se presenta en 19 a $45 \%$ de los sujetos con trastornos por consumo de sustancias, ${ }^{14,16}$ lo cual representa 2.6 veces el riesgo para la conducta suicida respecto de los sujetos que no los padecen. ${ }^{13} \mathrm{El}$ hallazgo de una mayor probabilidad de suicidio relacionado con el consumo de un mayor número de sustancias coincide con el estudio de Borges y colaboradores, quienes concluyen que más que el tipo de sustancia es el número de éstas el que predice la conducta suicida. ${ }^{9}$

Existen varias explicaciones para el incremento del riesgo de suicidio en las personas con trastorno por consumo de sustancias. En primer lugar, la teoría de la desinhibición propone que la intoxicación puede incrementar dicho riesgo al reducir la inhibición para cometer un acto impulsivo. ${ }^{9 ; 12}$ De la misma forma, tanto la intoxicación como la abstinencia de sustancias pueden alterar la capacidad de juicio y potenciar la desinhibición conductual. ${ }^{19}$

Las explicaciones alternativas guardan relación con la comorbilidad entre el trastorno depresivo mayor y el trastorno por consumo de sustancias cuya frecuencia es casi tres veces la de la población general. ${ }^{15}$ En este sentido, se ha encontrado que en personas con dependencia del alcohol hasta dos tercios de los que se suicidan presentan algún tipo de trastorno depresivo. ${ }^{19} \mathrm{Al}$ padecer ambos trastornos es posible llegar a un estado de escaso cuidado con la vida, con indiferencia y desesperanza. ${ }^{20}$ En este sentido, las sustancias adictivas pueden inducir modificaciones del estado de ánimo hacia la depresión y también hacia un estado de ánimo expansivo o irritable. ${ }^{19}$

Los hallazgos en relación con el alcohol como la sustancia con mayor porcentaje de detección en los casos 


\section{Cuadro IV \\ ANÁLISIS DE REGRESIÓN LOGÍSTICA ENTRE VARIABLES SOCIODEMOGRÁFICAS, número de sustancias detectadas y suicidio, Servicio Médico Forense 1994-2006, México}

\begin{tabular}{|c|c|c|c|c|}
\hline Variable & Hombres & & Mujeres & \\
\hline & $R M$ & IC95\% & $R M$ & IC95\% \\
\hline \multicolumn{5}{|l|}{ Edad } \\
\hline Menor de 20 & $2.16^{*}$ & $1.94-2.40$ & $3.56^{*}$ & $2.88-4.40$ \\
\hline 20 a 39 & $2.42 *$ & $2.26-2.60$ & $3.18 *$ & $2.70-3.74$ \\
\hline 40 o mayores & 1.00 & - & 1.00 & - \\
\hline \multicolumn{5}{|l|}{ Escolaridad } \\
\hline Primaria o menor & 1.00 & - & 1.00 & - \\
\hline Secundaria o mayor & 1.04 & $0.97-1.13$ & $1.34 *$ & I.14-1.57 \\
\hline \multicolumn{5}{|l|}{ Estado civil } \\
\hline Soltero & $1.19 *$ & $1.04-1.36$ & $1.43^{*}$ & $1.11-1.85$ \\
\hline Casado & $1.21^{*}$ & I.07-1.37 & $1.84 *$ & $|.46-2.3|$ \\
\hline Separado/divorciado/viudo & 1.00 & - & 1.00 & - \\
\hline \multicolumn{5}{|l|}{ Número de sustancias } \\
\hline Sin sustancias & 1.00 & - & 1.00 & - \\
\hline Una sustancia & $1.81^{*}$ & $1.69-1.93$ & $3.18 *$ & $2.66-3.79$ \\
\hline Dos o más sustancias & $3.30^{*}$ & $2.79-3.90$ & $3.61^{*}$ & I.83-7.II \\
\hline$* p<0.05$ & & & & \\
\hline
\end{tabular}

de suicidio bajo la influencia de sustancias coinciden con estudios previos, ${ }^{13,20-22}$ aunque en este estudio se encontró un porcentaje de $72.9 \%$, mayor al reportado por Pirkola y colaboradores, ${ }^{21}$ quienes en su estudio de revisión encontraron porcentajes de 15 a 56\%. En este sentido, cabe precisar que esta muestra estuvo constituida en mayor proporción por hombres, grupo que presenta con mayor frecuencia consumo de alcohol y fallecimiento por violencia (suicidio y homicidio). ${ }^{4,23}$

La relación entre el consumo de alcohol y el suicidio es compleja y parece incluir desde la alteración psicosocial hasta la desinhibición y la disforia, así como ser considerado un factor modificador entre los trastornos psiquiátricos y la conducta suicida. ${ }^{17,24-27}$ En este sentido, el aumento del consumo de alcohol puede propiciar más problemas físicos que pueden resultar en disfunción cerebral, cambios neuropsicológicos que incluyen deterioro del juicio, cambios del estado de ánimo y conducta violenta relacionada, ${ }^{17}$ factores que pueden potenciarse cuando existe el consumo de más de una sustancia.

Los hallazgos encontrados en las variables sociodemográficas que apuntaron a una mayor proporción de casos de suicidio bajo el influjo de sustancias en las mujeres y el grupo con mayor escolaridad son de llamar la atención. En este sentido, es probable que exista un subgrupo de mujeres con el antecedente de trastornos psiquiátricos comórbidos (trastorno depresivo mayor, trastorno por estrés postraumático, trastorno límite de la personalidad y dependencia de otras sustancias) que opten por intentar el suicidio con sobredosis de sustancias, como se ha notificado en otras publicaciones. ${ }^{13,28-32}$

Por otro lado, Agerbo ${ }^{33}$ encontró en la población danesa que el suicidio fue más frecuente entre los pacientes con ingresos altos y mayores logros académicos, en los casados y en los sujetos con empleo de tiempo completo. Este autor concluyó que es posible que un cambio rápido en el estatus social del individuo puede conducirlo a un estado de "disonancia social" y así elevar el riesgo para suicidio. Por otro lado, variables como el estado civil pueden registrarse de manera errónea ante pérdidas súbitas de la pareja.

En cuanto al hallazgo de este estudio en relación con una mayor frecuencia del suicidio bajo el influjo de sustancias en el grupo de edad de 20 a 39 años, históricamente se ha descrito que la tasa de suicidio es mayor en las personas mayores de 45 años. Sin embargo, en 
los últimos años se ha descrito un aumento de $30 \%$ de la frecuencia de suicidio en el grupo de edad de 25 a 34 años y en la actualidad la mayoría de los suicidios sucede en las edades de 15 a 44 años. Otro aspecto que podría explicar este hallazgo es que los sujetos con dependencia a alcohol y sustancias que cometen suicidio suelen tener entre 20 y 40 años. ${ }^{34,35}$

Los datos aquí obtenidos deben interpretarse con cautela, ya que el procedimiento para determinar el fallecimiento por suicidio y el tipo de sustancia pudo no ser el mismo en todos los casos. Asimismo, existe la posibilidad de que un número de muertes catalogadas como suicidios fuera en realidad producto de una sobredosis accidental, problema que se ha encontrado de manera frecuente en investigaciones sobre el suicidio en población con dependencia de sustancias. ${ }^{16,29}$ De acuerdo con Rossow y Lauritzen, ${ }^{20}$ el suicidio y la sobredosis son opuestos desde el punto de vista conceptual, ya que el primero es intencional y la segunda accidental. En este sentido, se han informado subgrupos de consumidores de sustancias con un nivel elevado de impulsividad, como el caso de los consumidores de heroína, en quienes se ha encontrado un rango de sobredosis anteriores a la conducta suicida de 48 a $68 \%{ }^{36}$

En conclusión, el consumo de sustancias es un factor importante relacionado con el suicidio al comparar con otras causas de defunción, en los sujetos cuya causa de defunción fue dictaminada por el SEMEFO de las entidades federativas participantes en el SISVEA. Sin embargo, deben establecerse mecanismos o metodologías más precisos para la detección de las sustancias con objeto de mejorar el registro de los casos de suicidio bajo la influencia de éstas.

\section{Agradecimientos}

Los autores agradecen la colaboración técnica del equipo que labora en el SISVEA de la DGAE: Dr. Armando Amezcua Jiménez, Lic. Otilda Jiménez Casillas, Lic. Maricela Mendoza Morales, C. Martha Villalobos Díaz y Dr. Juan Manuel Hernández Sánchez.

\section{Referencias}

I. Baca-García E, Aguilar E. Suicidio. En:Vallejo J, Leal C. Tratado de psiquiatría,Vol. II. Barcelona:Ars Médica, 2004:I446- 1457. 2. Díaz-Suárez J, Bousoño M, Bobes J. Concepto y clasificación de los comportamientos suicidas. En: Bobes J, Sáiz P, García-Portilla M, Bascarán M, Bousoño G. Comportamientos suicidas, prevención y tratamiento. Barcelona: Ars Médica, 2004: I- 10.

3.World Health Organization. Mental health: suicide prevention (SUPRE). [Consultado, 2008 enero]. Disponible en: http://www.who.int/mental_ health/prevention/suicide/suicideprevent/en/.
4. Instituto Nacional de Estadística, Geografía e Informática. Estadísticas de intentos de suicidio y suicidios de los Estados Unidos Mexicanos 2005. México: INEGI, 2008 [Consultado, 2008 enero]. Disponible en: http:// www.inegi.gob.mx/prod_serv/contenidos/espanol/biblioteca/. 5. Petronis K, Samuels J, Moscicki E, Anthony J. An epidemiologic investigation of potential risk factors for suicide attempts. Soc Psych Psy Epidemiol 1990;25:193-199.

6. Henriksson S, Suokas J, Isometsä E, Ostamo A, Lönnqvist J. Mental disorders and comorbidity in attempted suicide. Acta Psychiatr Scand 1996;94:234-240.

7. Cheng A, Mann A, Chan K. Personality disorder and suicide. $\mathrm{Br}$ J Psychiatry 1997; 170:44I-446.

8. Kessler R, Borges G,Walters E. Prevalence of and risk factors for lifetime suicide attempts in the National Comorbidity Survey. Arch Gen Psychiatry 1999;56:617-626.

9. Borges G,Walters E, Kessler R. Associations of substance use, abuse, and dependence with subsequent suicidal behavior.Am J Epidemiol 2000;151:781-789.

10. Hawton K, Houston K, Haw C, Townsend E, Harris L. Comorbidity of axis I and axis II disorders in patients who attempted suicide. Am J Psychiatry 2003;160:1494-1500.

II. Borges G,Wilcox H, Medina-Mora ME, Zambrano J, Blanco J,Walters E. Suicidal Behavior in the Mexican Comorbidity Survey (M-NCS): lifetime and I2-month prevalence, psychiatric factors and service utilization. Salud Mental 2005;28:40-47.

12. Bernal M, Haro JM, Bernet S, Brugha T, de Graaf R, Bruffaerts R, et al. Risk factors for suicidality in Europe: results from the ESEMED study.J Affect Disord 2007; I0I:27-34.

I3. Beautrais A, Joyce P, Mulder R, Fergusson D, Deavoll B, Nightingale S. Prevalence of comorbidity of mental disorders in persons making serious suicide attempts: a case-control study.Am J Psychiatry 1996; I53:1009-1014. 14. Bakken K,Vaglum P. Predictors of suicide attempters in substancedependent patients: a six-year prospective follow-up. Clin Pract Epidemiol Ment Health 2007; 10:3-20.

15. Agosti V, Levin F. One-year follow up study of suicide attempters treated for drug dependence. Am J Addictions 2006; 15:293-296.

16. Johnsson E, Fridell M. Suicide attempts in a cohort of drug abusers: a 5-year follow-up study. Acta Psychiatr Scand 1997;96:362-366.

17. Preuss U, Schuckit M, Smith T, Danko G, Bucholz K, Hesselbrock M, et al. Predictors and correlates of suicide attempts over 5 years in I,237 alcohol-dependent men and women. Am J Psychiatry 2002;160:56-53. 18. Tapia R, Kuri P, Cravioto P, Revuelta A, Cortés M. Manual para la vigilancia epidemiológica de las adicciones. $3^{\mathrm{a}}$ ed. México: Dirección General Adjunta de Epidemiología, Secretaría de Salud, 1999:14-26. 19. Santamarina S, Iglesias C,Alonso M. Comportamientos suicidas y consumo de sustancias psicotropas. En: Bobes J, Sáiz P, García-Portilla M, Bascarán M, Bousoño M. Comportamientos suicidas, prevención y tratamiento. Barcelona:Ars Médica, 2004:91-95.

20. Rossow I, Lauritzen G. Balancing on the edge of death: suicide attempts and life-threatening overdoses among drug addicts. Addiction 1999;94:209-219.

21. Pirkola S, Suominen K, Isometsa E. Suicide in alcohol- dependent individuals. Epidemiology and management. CNS Drugs 2004; 18:423-436 22. Roy A, Lamparski D, Dejong J, Moore V, Linnoila M. Characteristics of alcoholics who attempt suicide. Am J Psychiatry 1990; 147:76I-765. 23. Medina-Mora ME, Cravioto P,Villatoro J, Galván F, Fleiz C, Rojas E, et al. Encuesta Nacional de Adicciones 2002, Capítulo de Alcohol. Consejo Nacional contra las Adicciones, CONADIC, Instituto Nacional de Psiquiatría Ramón de la Fuente Muñiz, INPRFM, Dirección General de Epidemiología, DGE, Instituto Nacional de Estadística, Geografía e Informática. México: INEGI, 2002.

24. Hesselbrock M, Hesselbrock V, Symanski K, Weidenman M. Suicide Attempts and Alcoholism.J Stud Alcoh 1988;49:436-442. 
25. Driessen M,Veltrup C,Weber J, Ulrich J,Wetterling T, Dilling $H$. Psychiatric co-morbidity, suicidal ideation in alcoholics seeking treatment. Addiction 1998;93:889-894.

26. Preuss U, Schuckit M, Smith TL, Danko GP, Buckman K, Bierut L, et al. Comparison of 3190 alcohol-dependent Individuals with and without suicide attempts. Alcohol Clin Exp Res 2002;26:47I-477.

27. Conner K, Beautrias A, Conwell Y. Risk factors for suicide and medically serious suicide attempts among alcoholics: analyses of Canterbury Suicide Project Data.J Stud Alcohol 2003;64:55I-554. 28. Bradvik L, Frank A, Hulenvik P, Medvedeo A, Berglund M. Heroin Addicts reporting previous heroin overdoses also report suicide attempts. Suic Life-Threat Behav 2007;37:475-480.

29. Darke $S$, Ross J. Suicide among heroin users: rates, risk factors and methods. Addiciton 2002;97:1383-1394.

30. Maloney E, Degenhardt L, Darke S, Mattick R, Nelson E. Suicidal

Behaviour and associated risk factors among opiod-dependent individuals: a case-control study.Addiction 2007;102:1933-1941.
31. Roy A. Characteristics of cocaine-dependent patients who attempt suicide.Am J Psychiatry 200I; 158:1215-1219.

32. Roy A. Characteristics of opiate dependent patients who attempt suicide.J Clin Psychiatry 2002;63:403-407.

33. Agerbo E. High income, employment, postgraduate education, and marriage: a suicide cocktail among psychiatric patients. Arch Gen Psychiatry 2007;64:1377-1384.

34. Roy A. Suicide. In: Sadock BJ, Sadock VA. Comprehensive textbook of psychiatry. 7th ed., vol 2. Baltimore: Lippincot Williams \& Wilkins, 200I:203I.

35. Centers for Disease Control and Prevention (CDC). Suicide trends among young and young adults aged 10-24 years-United States, 19902004. MMWR Morb Mortal Wkly Rep 2007;56(35):905-908.

36. Baca C, Grant K. What heroin users tell us about overdose. J Addic Dis 2007;26:63-68 